\title{
The evolution and advances of biomarker use in clinical trials for breast cancer treatment-a narrative review
}

\author{
Jing He, Yijun Jia \\ AstraZeneca Oncology R\&D, Shanghai, China \\ Contributions: (I) Conception and design: J He; (II) Administrative support: J He; (III) Provision of study materials or patients: J He; (IV) Collection \\ and assembly of data: Y Jia; (V) Data analysis and interpretation: J He; (VI) Manuscript writing: All authors; (VII) Final approval of manuscript: All \\ authors. \\ Correspondence to: Jing He. 3F, No 199 Liangjing Rd, Zhangjiang Hi-tech Park, Shanghai 201203, China. Email: jing.he3@astrazeneca.com.
}

\begin{abstract}
Cancer is characterized with enormous heterogeneity, including intertumoral heterogeneity and intratumor heterogeneity, which represents the main hurdle and challenges for therapy. The great success in the precision medicine and personalized treatment among all cancer types has been noted in breast cancer. Treatment for breast cancer has evolved in the past decades starting from CMF regimen (cyclophosphamide, methotrexate and fluorouracil) chemotherapy as the standard of care regardless of histological type in 1970s to the current stage of molecular subtype-based therapy which can also be known as biomarker-driven therapy. In response to this great shift of paradigm, clinical trials in breast cancer have evolved during the past several decades, while biomarkers are becoming prominently valuable in driving breast cancer research and drug development. By enabling early detection, identifying treatment responders and monitoring treatment response and therapeutic effects, biomarker-based clinical trial design will speed the development process and allow evaluation of multiple patient groups while biomarker-driven personalized treatment approaches will lead to better patient outcomes. This review provides an overview of the biomarker landscape in different molecular subtypes of breast cancer and the use of prognostic biomarkers in assisting in predicting therapeutic outcomes and monitoring recurrence in patients with breast cancer.
\end{abstract}

Keywords: Biomarker; clinical trials; breast cancer; molecular subtypes; disease recurrence; therapeutic outcomes

Received: 04 December 2020; Accepted: 02 January 2021; Published: 31 January 2021.

doi: $10.21037 /$ tbcr-20-66

View this article at: http://dx.doi.org/10.21037/tbcr-20-66

\section{Introduction}

With the approval of newer drugs based on efficacy in patients stratified by the presence or absence of molecular biomarkers, we are in an era of precision medicine and biomarker guided therapy (1). Oncology, especially solid tumors, have been considered as a potential focus area for the development of biomarkers (2). This is substantiated by the increase in the number of Food and Drug Administration (FDA) approved drugs for oncologic indications that could be used for biomarker guided therapy. From 2006 to 2018, a total of 31 drugs have been approved for 38 oncologic indications by the FDA based on efficacy in patients with the presence or absence of a particular biomarker. Among them, 5 drugs, trastuzumab, lapatinib, pertuzumab, ado-trastuzumab emtansine and olaparib were approved for treating patients with breast cancer (3). This is further substantiated by the growing list of companion diagnostics approved by the FDA in which a total of 17 companion diagnostic assays have been approved for use in patients with breast cancer (4). This suggests that, among different types of cancers, breast cancer might represent the most robust type for the development of biomarker guided therapeutics and biomarker-based clinical trials.

The categorization of breast cancer into distinct histological and molecular subtypes had undergone paradigm shift in the last few decades. In 2003, the World 
Health Organization (WHO) categorized breast cancer into 17 histological types $(5,6)$. Since the histological categorization did not have any therapeutic implications, it was not subsequently used in clinical practice (7). Classification of breast cancer based on predictive biomarkers like expression of oestrogen receptor (ER), progesterone receptors (PR) and assessment of human epidermal growth factor receptor 2 (HER2) are clinically relevant for therapeutic decision making (8-10). Although breast cancers are frequently classified based on the hormone receptor (HR) (ER+ and/or $\mathrm{PR}+)$ and HER2 status, they are molecularly and histologically heterogenous (7) . Microarray based gene expression studies have expanded the scope of conventional molecular classification and had also established the correlation between molecular and histological types (11). Despite the availability of microarray based gene panels, immunohistochemistry (IHC) based identification of molecular subtypes (HR+ and/ or HER2+) is considered as gold standard (12). Cellular proliferation markers like Ki-67 which has been reported to be of prognostic value can also be considered as surrogate markers in clinical trials (13).

The previous approach to treatment of breast cancer is an "one size fits all" modality had undergone, and a paradigm shift towards a more personalized treatment based on genetic predisposing factors (14). Despite the identification of established biomarkers, the search for additional biomarkers for prediction of prognosis in breast cancer has not ceased. This is in part due to the newer targeted therapeutics, which by definition, performs better in distinct patient subgroups. The availability of biomarkers with good prognostic ability will help in selecting patient subgroups who could potentially benefit from a particular treatment regimen and shortening the drug development process. This also minimizes overtreatment, reduces the economic burden of unnecessary therapeutic regimens and improves quality of life in patients with breast cancer $(15,16)$. The purpose of this review is to provide an overview of the biomarker landscape in different molecular subtypes of breast cancer and the use of prognostic biomarkers updated information on the current status of prognostic biomarkers and their efficacy in assisting in predicting therapeutic outcomes and monitoring recurrence in patients with breast cancer. We present the following article in accordance with the Narrative reporting checklist (available at http://dx.doi. org/10.21037/tbcr-20-66).

\section{Biomarker landscape in different molecular subtypes of breast cancer}

\section{HER2 positive breast cancer}

Among patients with breast cancers, $15-30 \%$ of patients experience HER2 positive breast cancer which is known for its poor prognosis $(17,18)$. Trastuzumab is a landmark drug for the anti-HER2 treatment. Prior to the approval of trastuzumab, HER2 positive breast cancer was associated with high recurrence rates and poor survival outcomes (19-21). Trastuzumab was approved by the FDA in 1998 for the treatment of HER2 overexpressing, node positive or node negative breast cancer in adjuvant setting and for the systemic treatment of HER2 overexpressing metastatic breast cancer. It is recommended to be used either as a monotherapy or in combination with chemotherapy (22). Subsequent to the approval of trastuzumab, 7 other drugs targeting HER2 positive breast cancer have been approved by the FDA. Pertuzumab (HER2 targeted monoclonal antibody) (23), lapatinib (HER1 and HER2 tyrosine kinase inhibitor) (24), neratinib (HER tyrosine kinase inhibitor) (25), tucatinib (HER2 tyrosine kinase inhibitor) (26), trastuzumab emtansine (27) and trastuzumab deruxtecan (28) (antibody drug conjugate) were the currently available drugs targeting HER2 positive breast cancer. They change the treatment pattern and prognosis in HER2-positive breast cancer patients.

Until now, HER2 is still the only validated biomarker for the treatment with these targeted therapies. Trastuzumab has been evaluated both as monotherapy and in combination with chemotherapy in early and metastatic breast cancers. Owing to the mechanism of action of trastuzumab, it is effective only in patients with HER2 positive breast cancer. In early clinical trials with trastuzumab monotherapy, an overall response rate of $26 \%$ was observed which increased to $35 \%$ and $41 \%$ for patients with IHC defined HER2 levels of $3+$ and gene amplification by fluorescent in situ hybridization (FISH) confirmed HER 2 positive patients, respectively (29). Similarly, in patients with metastatic breast cancer, overall response rates of $26 \%$ was observed with significantly better response rate in patients with IHC3 + HER2 levels. Clinical benefit rates were also better for patients with higher HER2 expression levels (IHC3+ vs. IHC2+: $48 \%$ vs. $7 \%$ ). HER2 gene amplification by FISH also revealed similar improvement in efficacy for HER2 positive patients (29). These results suggest that, irrespective of HER2 detection method (FISH vs. IHC), 
patients with good HER2 levels most likely experiences a favorable therapeutic response to trastuzumab. Similar results were also observed with trastuzumab in combination with chemotherapy (30). Although the survival outcomes were significantly better in trastuzumab containing arm, a sub-population of patients seems to have no clinical benefit despite the presence of HER2 overexpression. A probable reason for the observed discrepancy with respect to the poor survival outcomes in a few patients could be the development of resistance to HER2 directed therapies (31).

Other molecular markers related to the HER2 pathway have also been evaluated for their role in predicting therapeutic response. The landmark Clinical Evaluation of Pertuzumab and Trastuzumab (CLEOPATRA) trial evaluated the efficacy of dual HER2 targeted therapy in combination with chemotherapy in HER2 positive, metastatic breast cancer patients (32,33). In an exploratory analysis from the CLEOPATRA trial, serum markers, ligands and receptors and intracellular pathway markers were assessed using appropriate assays to investigate their predictive therapeutic effects and prognostic effects independent of treatment arm. The biomarkers included amphiregulin, betacellulin, epidermal growth factor (EGF), transforming growth factor alpha, EGF receptor, HER2, HER3, insulin-like growth factor 1 receptor, PTEN, phosphorylated AKT, PIK3CA, CMYC, serum HER2 extracellular domain (sHER2), and FCR. These markers were estimated by IHC, FISH and reverse transcription polymerase chain reaction. Among the markers analyzed, high HER2 mRNA and HER3 mRNA, low sHER2 were significantly associated with a prolonged PFS. The analysis of prognostic effect of the biomarkers in the patients pooled from both the arms revealed PIK3CA mutation to be the most significant prognostic factor in patients with HER2 positive, metastatic breast cancer treated with either drug regimens. Patients with mutated PIK3CA had a worse PFS (8.6 months for mutated vs. 13.8 months for wild type in control group; 12.5 months for mutated vs. 21.8 months for wild type in pertuzumab group) (34). The identical results obtained in both the treatment groups in the CLEOPATRA trial suggested that mutation in PIK3CA could be a marker for HER2 resistance.

The efficacy of dual HER2 targeted therapy has also been evaluated in the neoadjuvant settings in the Chemotherapy, Herceptin and Lapatinib in Operable Breast Cancer (CHER-LOB) Trial. The trial evaluated the efficacy of chemotherapy in combination with trastuzumab or lapatinib and chemotherapy in combination with trastuzumab plus lapatinib in patients with HER2 positive, stage II to IIIA operable breast cancer. The addition of dual HER2 targeted drugs improved pCR rates In comparison to either drug alone (25\% vs. $26.3 \%$ vs. $46.7 \%$, $\mathrm{P}=0.019$ ) (35). In the subsequent predictive biomarker study, patients with wild type and mutated PIK3CA had similar pCR rates (33.7\% vs. $22.7 \%, \mathrm{P}=0.323)$ in the pooled cohort while PIK3CA mutated patients had a better pCR rate in patients treated with dual HER2 targeted therapy (48.4\% vs. $12.5 \%, \mathrm{P}=0.06$ ) (36). The similar rates of $\mathrm{pCR}$ among patients with PIK3CA wild type and mutated patients in this study could be due to the low number of PIK3CA mutated patients in the study $(n=5)$. However, the role of PIK3CA mutation in predicting resistance to HER2 directed therapies have been confirmed in multiple trials with single or dual HER2 directed therapies in neoadjuvant settings $(37,38)$. In a pooled stratified analysis of multiple trials with dual HER2 targeted therapy, patients with PIK3CA wild type have better pCR (45.5\% vs. $21.4 \%$ ) in comparison to patients with mutated PIK3CA (39).

The results of the above studies suggested a worse prognosis for patients with PIK3CA mutated, HER2 positive breast cancer in neoadjuvant, adjuvant and advanced metastatic settings. Hence inhibition of PI3K pathway might enhance clinical benefit in patients with HER2 positive breast cancer. The phase IB, PIKHER2 study (NCT01589861) evaluated the efficacy of a panisoform, class I, PI3K inhibitor, buparlisib, in combination with lapatinib in patients with HER2 positive, trastuzumab resistant, advanced breast cancer. The study reported a disease control rate of $79 \%$ (95\% CI, $57 \%$ to $92 \%$ ) and clinical benefit rate of $29 \%$ (95\% CI, $12 \%$ to $51 \%$ ) with one patient reporting complete response (40). In another similar phase II study (NCT01132664), buparlisib was evaluated in combination with trastuzumab in patients with trastuzumab resistant, HER2 positive, advanced breast cancer. The study reported an objective response rate (ORR) of $10 \%$ which was much lower than the predefined, primary endpoint (ORR $\geq 25 \%$ ). Among the 4 patients with partial response, 2 patients had PI3K pathway activated tumors (41). Nevertheless, both the studies did not select for the patients with PIK3CA mutation. Neoadjuvant PI3K inhibition in HER2 Over Expressing Breast cancEr (NeoPHOEBE) trial (NCT01816594), evaluated the efficacy of buparlisib or placebo in combination with trastuzumab and paclitaxel in patients with HER2 positive breast cancer in neoadjuvant setting. The study was terminated due to liver toxicity. But the premature results suggested a lack of enhanced efficacy 
with the addition of buparlisib with respect to the $\mathrm{pCR}$ rates ( $32 \%$ vs. $40 \%, \mathrm{P}=0.811$ ). However, in a small sub-group of ER+ patients, addition of buparlisib led to significant improvement in ORRs (68.8\% vs. 33.3\%, $\mathrm{P}=0.03$ ) (42).

The effectiveness of PI3K inhibition in combination with HER2 targeted therapy was evaluated in a phase I study (NCT02038010) with the $\alpha$-specific PI3K inhibitor, alpelisib. Alpelisib in combination with trastuzumab emtansine in trastuzumab resistant patients revealed an ORR of $43 \%$ which increased to $53 \%$ in patients with anomalies in the PI3K pathway. The clinical benefit rate was $56 \%$ in patients with mutation in any of the genes involved in the PI3K pathway including 3 patients who progressed on previous trastuzumab emtansine treatment (43). All the completed studies evaluating PI3K inhibition in combination with HER2 targeted drugs have included patients not selected for mutation in PI3K pathway which makes it difficult to interpret the precise role of $\mathrm{PI} 3 \mathrm{~K}$ mutation as a biomarker in selecting patients eligible for combination therapy with PI3K inhibitors and HER2 targeted drugs. An ongoing phase III study (NCT04208178) is currently evaluating the efficacy of alpelisib in combination with dual HER2 targeted therapy (trastuzumab and pertuzumab) in patients with HER2 positive, PIK3CA mutated, advanced breast cancer who have been previously treated with trastuzumab and pertuzumab. The results of this study will provide further insights on the prognostic value of PI3KCA mutation in predicting response to HER2 targeted drugs and for selecting patients for PI3K inhibitor treatment in the advanced breast cancer setting.

Biomarkers of immunity may also have potential prognosis significance in HER2-positive advanced breast cancer. A retrospective analysis of the CLEOPATRA study assessed the prognostic ability of tumor infiltrating lymphocytes (TILs) in predicting therapeutic outcomes. TILs from the available tumor samples were assessed by histopathology and a cut-off of $20 \%$ stromal TILs were used for stratified regression analysis. The median PFS values were similar in patients with TILs $\leq 20 \%$ and $>20 \%$ (HR: 0.85 ; 95\% CI, 0.70 to 1.03 ) whereas median OS was significantly shorter in patients with TIL $\leq 20 \%$ (HR 0.76 ; $95 \%$ CI, 0.60 to 0.96 ). However, there was significant interaction between stromal TIL values and drug treatment with respect to both PFS and OS (44). This suggests that stromal TIL might be potential prognostic marker for predicting OS in patients with HER2 positive, metastatic breast cancer irrespective of the treatment regimen. This finding has also been substantiated in a recent meta-analysis of 5 randomized controlled trials involving patients with
HER2 positive, non-metastatic breast cancer. The pooled odds of pathologic complete remission (pCR) in high TIL expressing patients from 4 studies involving trastuzumab as the only HER2 targeted agent, in combination with chemotherapy, was found to be 1.90 (95\% CI, 1.21 to 3.00). Similar results were also obtained from studies with lapatinib as the sole HER2 targeted agent [odds ratio (OR): $1.79,95 \%$ CI, 0.99 to 3.23]. Surprisingly, despite the lack of interaction between treatment arms and TIL expression in predicting pCR, studies with dual HER2 targeted therapies revealed a higher odds of pCR in high TIL expressing patients (OR: 3.04; 95\% CI, 0.84 to 11.20), although there was no statistical significance $(\mathrm{P}=0.147)(45)$.

\section{HR positive $(H R+)$ breast cancer}

Hormone receptors including ER and PR are expressed in approximately $80 \%$ of breast cancer (46). Owing to the expression of HRs, endocrine therapy has been the mainstay of treatment in patients with HR positive breast cancer in neoadjuvant, adjuvant and advanced treatment settings (29). Determination of HR status is mandatory for selecting patients who are likely to respond to endocrine therapies like the selective ER modulators (tamoxifen), aromatase inhibitors (anastrozole, letrozole and exemestane), luteinising-hormone releasing hormone agonists (goserelin), selective ER degrader (fulvestrant) and oophorectomy, across different treatment settings (47-49). The prognostic ability of HR in predicting response to endocrine therapies have been reported in multiple studies. In a patient level meta-analysis on the efficacy of adjuvant tamoxifen, the relative risk (RR) for disease recurrence was significantly low for up to 10 years in patients with ER positive breast cancer (RR: $0.62, \mathrm{P}<0.00001)$. In patients with ER positive breast cancer, PR status was not predictive factor for disease recurrence (10). This suggests that there could be more heterogeneity with respect to predictive biomarkers with other genetic factors also influencing the disease free survival in patients with HR positive disease (50-52). Multiple retrospective studies have reported superior outcome in ER or PR positive tumors. But the observed favourable outcome seems to be negated after $5-10$ years of adjuvant treatment $(51,52)$. This suggests that HR receptor status alone cannot predict therapeutic outcome in patients with HR positive, early breast cancer, treated with endocrine therapies. In a previous retrospective study, patients with PIK3CA mutation had a better PFS 
when treated with everolimus (53). To address the issue, the updated guidelines from the European group on tumor markers recommended future research focusing on the development of biomarkers with increased positive predictive value and those that could assist in selection of patients who could potentially benefit from specific endocrine therapy (54).

Patients with HR positive breast cancer respond differentially with temporal variation to endocrine therapy which suggest role of other molecular pathways especially the ones involved in cell cycle. Cyclin-dependent kinases $(\mathrm{CDK}) 4$ and 6 promote cell cycle entry and in vitro studies confirm their role in breast cancer proliferation $(55,56)$. Further in vitro studies reported that breast cancer resistant to endocrine therapy might still be dependent on CDK4/6 (57,58). Based on these observations, 3 CDK4/6 inhibitors, palbociclib, ribociclib and abemaciclib have been approved by the FDA for the treatment of patients with HR positive, HER2 negative, early and metastatic breast cancer (59-61). The approval of CDK4/6 inhibitors has changed the treatment landscape of patients with HR positive breast cancer. CDK4/6 inhibitors have been used in combination with different endocrine therapy regimens like aromatase inhibitors, fulvestrant and tamoxifen and the probable biomarkers predicting therapeutic outcome have been evaluated in the landmark trials.

In the PALOMA-3 trial (NCT01942135), fulvestrant in combination with palbociclib was found to be effective in prolonging PFS in the overall patients and also in stratified subgroups in the advanced treatment settings. The combination was found to be equally effective in both $\mathrm{ER}+/ \mathrm{PR}$ - and ER+/PR+ subgroups (HR: 0.46 for both the subgroups) (46). Further, the study also reported that a subset of patients treated with fulvestrant alone elucidated a prolonged progression free survival (62). Subsequently, an exploratory biomarker analysis was performed. The predictive ability of mutations in PI3KCA and the gene encoding the main endoplasmic reticulum isoform (ESR1) was assessed in long-term ( $\geq 18$ months) and short-term responders (<18 months). The incidence of ESR1 and PI3KCA mutation at baseline was lower in long-term responders in both the treatment arms. However, a more pronounced difference in mutation rates were observed in the fulvestrant plus placebo arm. The incidence of ESR 1 mutation was $6 \%$ and $33 \%$ in long-term and shortterm responders while the corresponding incidence of PI3KCA mutation was $6 \%$ and $39 \%$ in long- and shortterm responders, respectively (63). This suggested that the baseline mutation in these genes may attenuate the resistance to endocrine therapy and by extension, the survival outcomes in patients with $\mathrm{HR}+, \mathrm{HER} 2-$ locally advanced/metastatic breast cancer. In a pooled analysis of two different trials, patients with ESR1 mutation and ESR1 wild type in the PALOMA-3 trial reported better PFS when treated with palbociclib in combination with fulvestrant than fulvestrant alone (HR: 0.43 for ESR1 mutated and 0.49 for wild type, $\mathrm{P}<0.05$ for both). Stratified analysis from the SoFEA study evaluating fulvestrant and exemestane revealed that patients with ESR1 mutation had a significantly better PFS (HR: 0.52, $\mathrm{P}=0.02$ ) when treated with fulvestrant which corroborated with the exploratory analysis of the PALOMA-3 study (64-66). This suggested that ESR1 mutation could be used to select patients for treatment with fulvestrant over aromatase inhibitors. An analysis stratified by molecular subtype of breast cancer (luminal A vs. luminal B) in patients treated in the PALOMA-3 trial revealed similar effect estimates for treatment with fulvestrant plus palbociclib (HR for PFS for luminal A and B: 0.23 and $0.26, \mathrm{P}<0.0001$ for both). Baseline mRNA levels of CDK6 and cyclin E1 (CCNE1) were significantly associated $(\mathrm{P}<0.05$ for both) with treatment outcome in patients with bone only and visceral only metastases, respectively. CCNE1 was also associated significantly with therapeutic outcomes in patients with non-visceral (excluding bone) metastasis in patients analysed in the PALOMA-3 trial (29). In another study employing paired baseline and end of study sequencing of ctDNA, from the PALOMA-3 study, newer driver mutations in the PIK3CA and ESR 1 genes emerged during treatment in both the arms (67). This suggested that mutations in these 2 loci might have therapeutic implications for treatment with other endocrine drugs like aromatase inhibitors. The clinical significance of these mutations with respect to stage of disease and line of treatment was assessed by a study by Takeshita et al. They analysed the ctDNA of early and metastatic BC patients and identified that the prevalence of mutations in $P I K 3 C A$ and ESR 1 genes were high in late treatment settings. This suggested the role of endocrine therapy in driving the mutations leading to resistance to endocrine therapy. The same study also revealed shorter time to treatment failure in PIK3CA mutated patients treated at early lines of treatment while there was no statistically significant difference in patients with ESR1 mutations (68). Hence the current evidence suggests a role for mutations in PIK3CA and ESR1 genes to impact treatment outcomes with endocrine therapy. This should be considered while designing future trials to 
evaluate the efficacy of drugs with similar molecular targets.

The PALOMA-2 trial (NCT01740427) evaluated the efficacy of the aromatase inhibitor, letrozole in combination with palbociclib in comparison to monotherapy with letrozole. The study revealed superior efficacy of letrozole in combination with palbociclib (HR for PFS: 0.58, $\mathrm{P}<0.001)$ as against therapy with letrozole alone (69). In a extensive biomarker analysis of the PALOMA-2 study, subgroups stratified by above and below median expression of multiple genes at baseline was performed. The results revealed significant improvement in PFS in patients treated with letrozole and palbociclib irrespective of the basal mRNA levels of all the analysed genes. Whereas, changes in expression of CDK4 significantly altered the PFS in patients treated with letrozole alone. This substantiated the role of CDK4 expression as a prognostic marker for resistance to endocrine therapy. Similarly high ESR1 expression was associated with better PFS irrespective of treatment groups. But the addition of palbociclib seems to bring benefits to patients with both low and high ESR1 expression. Molecular subtype of breast cancer (luminal A $v s$. B) was also not associated with differential treatment effect. Extensive analysis with large gene panels revealed only increased expression of fibroblast growth factor receptor 2 (FGFR2) ( $\mathrm{P}=0.05)$, Erb-B2 receptor tyrosine kinase $3(\mathrm{ERBB} 3)(\mathrm{P}=0.07)$ and programmed cell death protein $1(\mathrm{PD}-1)(\mathrm{P}=0.024)$ to be associated with $\mathrm{PFS}$ in patients treated with palbociclib and letrozole (70). In a statistically adjusted analysis of the PALOMA-2 trial, the expression of $\mathrm{CDK} 4$ was associated with treatment outcome only in patients with visceral metastases $(\mathrm{P}=0.010)(71)$.

The results of the biomarker analysis from PALOMA-2 and PALOMA-3 studies did not reveal conclusive findings with respect to patients who may derive more benefits from the addition of CDK4/6 inhibitors to endocrine therapy in HR+, HER2- advanced breast cancer. Further exploration of biomarkers which can predict therapeutic effects and with prognostic value in patients treated with endocrine therapy is warranted. Similar to HER2 positive breast cancer, triple therapy with PI3K inhibitors like alpelisib, endocrine therapy and CDK4/6 inhibitors is also being explored in patients with HR +, HER2-, PI3K mutations $(72,73)$.

\section{Triple negative breast cancer (TNBC)}

Among the molecular types of breast cancer, TNBC constitutes the most heterogenous tumor type which is difficult to be treated because of the difficulties in finding the targeted therapies with current available therapeutic options (74). Unlike patients with HR positive and HER2 positive breast cancer, the molecular mechanism that drives the development of cancerous cell mass has not been identified in TNBC $(75,76)$. Based on genomic expression profiles, TNBC is classified into 4 broad types, namely, basal-like subtype 1 , basal-like subtype 2, mesenchymal (M) and luminal androgen receptor (LAR), which also has therapeutic implications (77). Based on gene expression profiling, Lehmann et al. reported that $41 \%$ of patients with the BL1 subtype experienced pCR while $18 \%$ and $29 \%$ of patients with BL2 and LAR subtype experienced pCR after treatment with neoadjuvant chemotherapy (77).

Among the different cellular proteins, the tumor protein gene 53 (TP53) has been assessed for its prognostic ability in previous studies. In a study by Kim et al., TNBC patients with missense mutation in the TP53 gene had poor 5-year distant recurrence free survival. In another retrospective study, TNBC patients with detectable TP53 by IHC had higher OS than patients without TP53 expression $(78,79)$. The observational nature of these studies may have introduced confounding biases which needs to be addressed in prospective studies. Although previously considered as a difficult therapeutic target, TP53 has been explored as a drug target in invitro studies with TP53 mutated cell lines which were significantly more sensitive to the investigative drug, PRIMA-1 and PRIMA-1MET (80).

Although numerous other biomarkers have been found to be differentially expressed in patients with TNBC, none of the biomarkers have been established to be associated with therapeutic outcomes. Even cell proliferation related prognostic markers like $\mathrm{Ki}-67$ have been reported to be of limited value in patients with TNBC $(74,81)$. A main reason for the lack of prognostic biomarkers is the absence of well-defined targeted therapies. Among the available therapeutic options, apart from chemotherapy, poly (ADP-ribose) polymerase (PARP) inhibitors (PARPi) are a promising option for therapy for patients with TNBC (82). Germline mutation in BRCA1 gene has been reported to be a predisposing factor for TNBC and olaparib, a PARPi, has been reported to be effective in BRCA1/2 mutated TNBC patients (83). In the pivotal phase 3 trial (NCT02000622) that evaluated the efficacy and safety of olaparib in comparison to standard of care treatment in patients with germline mutation in BRCA1/2, olaparib exhibited superior efficacy. The study was performed in metastatic setting. The overall HR for PFS was 0.58 (95\% CI, 0.43 to 0.80 ) which 
was improved to 0.43 (95\% CI, 0.29 to 0.63$)$ in TNBC patients as against 0.82 (95\% CI, 0.55 to 1.26$)$ in HR positive patients. Similarly, patients with BRCA1 mutation had a significantly better HR when treated with olaparib in comparison to patients BRCA2 mutated patients (HR of $0.54, \mathrm{P}<0.05$ vs. HR of $0.68, \mathrm{P}>0.05)$ (84). A subsequent sub-group analysis of patients with TNBC and BRCA1 mutation has not been published yet. But the observed improvement in $\mathrm{HR}$ in TNBC and BRCA1 mutated patients suggests that BRCA1 mutation could be a potential prognostic biomarker in patients with TNBC treated with PARPi.

A previous registry-based real-world study (PRAEGNANT registry) from Germany in HER2 negative patients in metastatic setting reported a lack of significant association between BRCA status and prognosis after treatment with chemotherapy in patients with TNBC (85). This suggests that BRCA mutation may not be significant independent prognostic factor for patients with TNBC, but could be used to select for patients who could derive potential benefit from PARPi treatment.

In a recent abstract presented at the annual meeting for European Society for Medical Oncology, the effectiveness of olaparib monotherapy in neoadjuvant treatment of patients with TNBC was reported. As per the study, the ORR in overall patients was 64\% (5 complete response and 15 partial response), while in patients with somatic or germline BRCA1/2 mutations, the ORR was $100 \%$ (3/5 complete response and $2 / 5$ partial response). All the non-responders were negative for all known mutations in the DNA damage repair genes (ATRX, BRCA1/2, EMSY, MSH6, PARP10, PPM1D) and mutation in TP53 was not associated with response to olaparib (86).

Among other potential targeted therapy regimens in TNBC, the use of immune checkpoint inhibitors, especially programmed cell death 1 (PD-1) and programmed cell death-ligand 1 (PD-L1) seems to be promising. The IMpassion 130 phase 3 clinical trial (NCT02425891) evaluated the efficacy of atezolizumab, a PD-L1 inhibitor, with nab-paclitaxel in TNBC patients with metastatic disease. The study reported a statistically non-significant OS advantage for atezolizumab in combination with nab-paclitaxel (HR:0.86, 85\% CI:0.73 to 1.02$)$. But in the PD-L1 expression positive patients, the OS significantly favoured atezolizumab (HR: 0.71, $95 \% \mathrm{CI}, 0.54$ to 0.94$)$ (87). PD-L1 expression which is considered as a surrogate marker for the prediction of PD-1/PD-L1 inhibitor efficacy in other cancer types, could also be considered in TNBC. The issues pertaining to PDL1 expression testing like testing platform, desired target cell population (immune cells $v s$. tumor cells), threshold and antibody clones needs to be resolved for clinical decision making. In the biomarker exploratory analysis of the IMpassion study, PD-L1 expression in the tumor cells and the immune cells were separately evaluated. Patients with PD-L1 positive tumor cells had a relatively better PFS (HR of 0.62 in immune cells $v$ s. 0.51 in tumor cells) and similar OS (HR of 0.62 in immune cells $v s .0 .63$ in tumor cells) in comparison to immune cell defined PD-L1 positivity. CD8 $\geq 0.5$ (HR for PFS: 0.74 , HR for OS: $0.66, \mathrm{P}<0.05$ for both) and TILs $\geq 10 \%$ (HR for PFS: $0.66, \mathrm{P}<0.05$; HR for OS:0.75, P>0.05) were also associated with improvement in PFS and OS (88). But the clinical utility of TILs and CD8 levels require further studies for confirmation.

Atezolizumab has also been evaluated as monotherapy in patients with metastatic TNBC. In a early phase clinical study, treatment with atezolizumab led to stable clinical response and patients with $\mathrm{PD}-\mathrm{L} 1$ positive expression had higher ORR (12\%) and OS (10.1 months). High levels of tumor infiltrating immune cells (>10\%) was an independent factor predicting objective response and longer OS (89). The phase II KEYNOTE-086 study evaluated the effectiveness of pembrolizumab in PD-L1 positive patients with metastatic TNBC. The study reported a moderate ORR (21.4\%), PFS (2.1 months) and OS (18 months) (90). Despite selecting patients for PD-L1 expression, the lower response rates and $O S$ in metastatic setting suggest a limited role for PD-L1 in predicting therapeutic outcome with PD-1/PD-L1 drugs in metastatic TNBC.

Apart from the above studies, capivasertib, a kinase inhibitor with activity against PI3K/AKT activated tumors was tested in metastatic TNBC patients. There was no statistically significant improvement in PFS and OS in the overall patients. But in the sub-group of patients with PIK3CA/AKT1/PTEN-altered tumors, PFS was significantly better for capivasertib (HR, 0.30; 95\% CI, 0.11 to 0.79) (91). Similarly, ipatasertib, an AKT inhibitor, in combination with paclitaxel, also improved PFS in comparison to paclitaxel alone (HR: 0.60, 95\% CI, 0.37 to 0.98) (92). Similarly, MK-2206, which is an allosteric AKT inhibitor, has been evaluated in a recent phase-2 study. The study revealed limited clinical activity for MK-2206 in patients with PIK3CA/AKT1 mutation (ORR: 5.6\%; 1/18 partial response) and PTEN loss/mutation (ORR: 0\%). At present, three different clinical studies are in progress, and it is expected that satisfactory curative effect may be 
obtained in biomarker-defined subgroups.

\section{Biomarker landscape in early detection of recurrence}

Early detection of disease relapse before overt recurrence assist in prompt change in therapeutic intervention to extend the disease free survival. It could also improve the ability of biomarkers to predict outcome with subsequent therapeutic regimens. Among the available biomarkers for predicting recurrence, circulating tumor DNA (ctDNA) had been reported to predict recurrence providing sufficient lead time for changing therapeutic regimen. A multicenter prospective study, published in Clinical Cancer Research in 2019, monitored the disease recurrence of patients by analysing ctDNA. The study included 49 breast cancer patients who completed surgery and adjuvant therapy, including 34 patients with $\mathrm{HR}+/ \mathrm{HER} 2-, 8$ patients with HR+/HER2+, and 7 patients with TNBC. During the whole study period, 18 patients relapsed and 31 patients remained disease free. The plasma samples were estimated for the presence of ctDNA every 6 months by ultra deep sequencing. A total of 208 plasma samples were obtained, and the results showed that of the 18 patients with recurrence, 16 had positive ctDNA before clinical or imaging diagnosis of recurrence. In HR+/HER2- breast cancer patients, the lead time to report recurrence was 301 days before clinical diagnosis of recurrence. However, HR+/HER2+ breast cancer patients reported that the lead time to detect early recurrence was 164 days and that of TNBC was 258 days (93). The insights provided by this study suggest that ctDNA detection could be incorporated into routine clinical practice for delaying progression in early breast cancer patients. But the practical challenges might include determining the optimum frequency, timing, threshold of ctDNA and sensitivity of the testing platform, for clinical practice. Nevertheless, future studies could provide further insights on the utility of determining ctDNA levels.

Apart from detecting early recurrence, ctDNA could also monitor drug efficacy in real-time. The PALOMA 3 trial that evaluated the efficacy of palbociclib in combination with fulvestrant also reported the probable genetic markers for predicting early recurrence. The study reported higher baseline tumor purity (HR: 1.20), baseline TP53 (HR: 1.84), baseline FGFR1 amplification (HR: 2.91) to be associated with worse PFS (67). In another exploratory study from the PALOMA 3 trial, ctDNA analysis was performed during the treatment process from day 1 to day 15 . The results showed that the median PFS was only 4.1 months in patients with less decline in PIK3CA ctDNA within 15 days, while the median PFS of patients with more change in PIK3CA ctDNA was 11.2 months. One group of patients with low circulating DNA ratio (CDR) (the ratio of ctDNA on the $15^{\text {th }}$ day to the $1^{\text {st }}$ day) can continue to receive palbociclib treatment, while the other group of patients with higher CDR could be treated with a different therapeutic regimen (94). This study suggests that changes in ctDNA on day 15 can help to identify those patients who may potentially benefit from palbociclib early and change treatment options for those who may not experience favourable treatment outcome after treatment with palbociclib and fulvestrant. Based on the results of this study, a possible future clinical study design can be made to stratify patients by ctDNA level on the $15^{\text {th }}$ day.

The difference in genetic mutation landscape within subclonal cell populations needs further studies for providing conclusive results. In RCTs, mutation landscape is either assessed in stored tumor samples or in ctDNA both of which may not provide the complete mutation landscape. This could be the reason for outliers in exploratory studies and in observational studies evaluating the role of mutations on the therapeutic outcomes. In future, patients may have to be assessed for mutation periodically from ctDNA for improving the clinical care. But assessment of mutation landscape in metastatic sites and continuous monitoring of tumor samples may not be practically possible.

\section{Biomarkers landscape in therapeutic outcome prediction}

Long-term exposure to therapeutic doses of chemotherapeutic drugs may lead to severe adverse events. With the usage of combination chemotherapy and targeted therapy, de-escalation of chemotherapy could be achieved without reducing therapeutic outcome. Biomarkers could also assist in selecting patients for appropriate de-escalation therapy. In case of HER2 positive breast cancer, dual HER2 targeted therapy in combination with chemotherapy is the standard of care in metastatic breast cancer. The PerELISA study evaluated Ki-67 guided usage of dual HER2 targeted therapy (trastuzumab and pertuzumab) in combination with letrozole and chemotherapy (paclitaxel). The patients (HR positive, HER2 positive) were tested after 2 weeks of letrozole treatment. Patients whose Ki-67 level decreased by more than $20 \%$ relative to baseline (molecular remission) continued treatment with trastuzumab 
+ pertuzumab + letrozole, while patients without molecular response were treated with paclitaxel (13 weeks) + trastuzumab + pertuzumab for one week. The results showed that $20.5 \%$ of patients with molecular remission achieved pCR, while $81.3 \%$ of patients with molecular non-response achieved pCR with additional chemotherapy. In addition, PAM50 subtypes were significantly associated with $\mathrm{Ki}-67$ levels and pCR. In patients with molecular remission, the pCR of HER2-enriched breast cancer was significantly higher than that of non-HER2 enriched patients (45.5\% vs. $13.8 \%, \mathrm{P}=0.042$ ) (95). This study suggests that $\mathrm{Ki}-67$ levels could assist in selecting patients for de-escalation of chemotherapy in patients with $\mathrm{HR}+$, HER2+ breast cancer. However, the study recruited a relatively small number of patients and did not have any comparator. Hence the precise role of the additional chemotherapy regimen is still uncertain.

\section{Conclusions}

In oncology specifically breast cancer, the posture toward biomarker development for innovative therapies is becoming the norm versus exception which will be adopted to this evolving paradigm of precision medicine and personalized therapy. A shift has been brought to the design and conduct of clinical trials in breast cancer by the current era of targeted therapy. Understanding the biomarker landscape, inluding the clinical research activity, is critical in drug development. This new knowledge will help us classify patients into even smaller subgroups, customize treatment, monitor efficacy, predict therapeutic outcome based on their specific biomarkers, leading to a brand new drug development paradigm.

\section{Acknowledgments}

This work was supported by Dr. G. Kaushik Subramanian and Dr. Amit Bhat, Indegene Pvt Ltd. who provided the medical writing assistance under the guidance of authors. Funding: None.

\section{Footnote}

Reporting Checklist: The authors have completed the Narrative reporting checklist. Available at http://dx.doi. org/10.21037/tbcr-20-66

Peer Review File: Available at http://dx.doi.org/10.21037/ tbcr-20-66
Conflicts of Interest: Both authors have completed the ICMJE uniform disclosure form (available at http://dx.doi. org/10.21037/tbcr-20-66). The authors have no conflicts of interest to declare.

Ethical Statement: The authors are accountable for all aspects of the work in ensuring that questions related to the accuracy or integrity of any part of the work are appropriately investigated and resolved.

Open Access Statement: This is an Open Access article distributed in accordance with the Creative Commons Attribution-NonCommercial-NoDerivs 4.0 International License (CC BY-NC-ND 4.0), which permits the noncommercial replication and distribution of the article with the strict proviso that no changes or edits are made and the original work is properly cited (including links to both the formal publication through the relevant DOI and the license). See: https://creativecommons.org/licenses/by-nc-nd/4.0/.

\section{References}

1. Cutler DM. Early Returns From the Era of Precision Medicine. JAMA 2020;323:109-10.

2. Collins FS, Varmus H. A New Initiative on Precision Medicine. N Engl J Med 2015;372:793-5.

3. Marquart J, Chen EY, Prasad V. Estimation of the Percentage of US Patients With Cancer Who Benefit From Genome-Driven Oncology. JAMA Oncol 2018;4:1093-8.

4. Health C for D and R. List of Cleared or Approved Companion Diagnostic Devices (In Vitro and Imaging Tools). FDA [Internet] 2020 Nov 16 [cited 2020 Nov 24]; Available online: https://www.fda.gov/medical-devices/ vitro-diagnostics/list-cleared-or-approved-companiondiagnostic-devices-vitro-and-imaging-tools

5. Weigelt B, Reis-Filho JS. Histological and molecular types of breast cancer: is there a unifying taxonomy? Nat Rev Clin Oncol 2009;6:718-30.

6. Tavassoli FA, Devilee P. WHO Classification of Tumours. Pathology and Genetics of Tumours of the Breast and Female Genital Organs. Lyon Press, Lyon; 2003.

7. Guiu S, Michiels S, André F, et al. Molecular subclasses of breast cancer: how do we define them? The IMPAKT 2012 Working Group Statement. Ann Oncol 2012;23:2997-3006.

8. Hammond MEH, Hayes DF, Dowsett M, et al. American Society of Clinical Oncology/College of 
American Pathologists guideline recommendations for immunohistochemical testing of estrogen and progesterone receptors in breast cancer (unabridged version). Arch Pathol Lab Med 2010;134:e48-72.

9. Wolff AC, Hammond MEH, Schwartz JN, et al. American Society of Clinical Oncology/College of American Pathologists guideline recommendations for human epidermal growth factor receptor 2 testing in breast cancer. Arch Pathol Lab Med 2007;131:18-43.

10. Early Breast Cancer Trialists' Collaborative Group (EBCTCG), Davies C, Godwin J, et al. Relevance of breast cancer hormone receptors and other factors to the efficacy of adjuvant tamoxifen: patient-level meta-analysis of randomised trials. Lancet 2011;378:771-84.

11. Sørlie T, Perou CM, Tibshirani R, et al. Gene expression patterns of breast carcinomas distinguish tumor subclasses with clinical implications. Proc Natl Acad Sci U S A 2001;98:10869-74.

12. Goldhirsch A, Wood WC, Coates AS, et al. Strategies for subtypes--dealing with the diversity of breast cancer: highlights of the St. Gallen International Expert Consensus on the Primary Therapy of Early Breast Cancer 2011. Ann Oncol 2011;22:1736-47.

13. Inwald EC, Klinkhammer-Schalke $M$, Hofstädter F, et al. $\mathrm{Ki}-67$ is a prognostic parameter in breast cancer patients: results of a large population-based cohort of a cancer registry. Breast Cancer Res Treat 2013;139:539-52.

14. Harris EER. Precision Medicine for Breast Cancer: The Paths to Truly Individualized Diagnosis and Treatment [Internet]. International Journal of Breast Cancer 2018 [cited 2020 Nov 25]. Available online: https://www. hindawi.com/journals/ijbc/2018/4809183/

15. Monticciolo DL, Helvie MA, Hendrick RE. Current Issues in the Overdiagnosis and Overtreatment of Breast Cancer. AJR Am J Roentgenol 2018;210:285-91.

16. Stopeck AT, Brown-Glaberman U, Wong HY, et al. The role of targeted therapy and biomarkers in breast cancer treatment. Clin Exp Metastasis 2012;29:807-19.

17. Siegel RL, Miller KD, Jemal A. Cancer statistics, 2019. CA Cancer J Clin 2019;69:7-34.

18. Derakhshani A, Rezaei Z, Safarpour H, et al. Overcoming trastuzumab resistance in HER2-positive breast cancer using combination therapy. J Cell Physiol 2020;235:3142-56.

19. Press MF, Pike MC, Chazin VR, et al. Her-2/neu expression in node-negative breast cancer: direct tissue quantitation by computerized image analysis and association of overexpression with increased risk of recurrent disease. Cancer Res 1993;53:4960-70.

20. Tiwari RK, Borgen PI, Wong GY, et al. HER-2/neu amplification and overexpression in primary human breast cancer is associated with early metastasis. Anticancer Res 1992;12:419-25.

21. Slamon DJ, Godolphin W, Jones LA, et al. Studies of the HER-2/neu proto-oncogene in human breast and ovarian cancer. Science. 1989;244:707-12.

22. Highlights of prescribing information, trastuzumab. Food and Drug Administration; Report No.: U.S. BL 103792.

23. Swain SM, Kim SB, Cortés J, et al. Pertuzumab, trastuzumab, and docetaxel for HER2-positive metastatic breast cancer (CLEOPATRA study): overall survival results from a randomised, double-blind, placebo-controlled, phase 3 study. Lancet Oncol 2013;14:461-71.

24. Geyer CE, Forster J, Lindquist D, et al. Lapatinib plus capecitabine for HER2-positive advanced breast cancer. N Engl J Med 2006;355:2733-43.

25. Chan A, Delaloge S, Holmes FA, et al. Neratinib after trastuzumab-based adjuvant therapy in patients with HER2-positive breast cancer (ExteNET): a multicentre, randomised, double-blind, placebo-controlled, phase 3 trial. Lancet Oncol 2016;17:367-77.

26. Murthy RK, Loi S, Okines A, et al. Tucatinib, Trastuzumab, and Capecitabine for HER2-Positive Metastatic Breast Cancer. N Engl J Med 2020;382:597-609.

27. Diéras V, Miles D, Verma S, et al. Trastuzumab emtansine versus capecitabine plus lapatinib in patients with previously treated HER2-positive advanced breast cancer (EMILIA): a descriptive analysis of final overall survival results from a randomised, open-label, phase 3 trial. Lancet Oncol 2017;18:732-42.

28. Modi S, Saura C, Yamashita T, et al. Trastuzumab Deruxtecan in Previously Treated HER2-Positive Breast Cancer. N Engl J Med 2020;382:610-21.

29. Vogel CL, Cobleigh MA, Tripathy D, et al. Efficacy and safety of trastuzumab as a single agent in first-line treatment of HER2-overexpressing metastatic breast cancer. J Clin Oncol 2002;20:719-26.

30. Romond EH, Perez EA, Bryant J, et al. Trastuzumab plus adjuvant chemotherapy for operable HER2-positive breast cancer. N Engl J Med 2005;353:1673-84.

31. Choong GM, Cullen GD, O'Sullivan CC. Evolving standards of care and new challenges in the management of HER2-positive breast cancer. CA Cancer J Clin 2020;70:355-74.

32. Swain SM, Baselga J, Kim SB, et al. Pertuzumab, Trastuzumab, and Docetaxel in HER2-Positive Metastatic 
Breast Cancer. N Engl J Med 2015;372:724-34.

33. Nahta R, Hung MC, Esteva FJ. The HER-2-targeting antibodies trastuzumab and pertuzumab synergistically inhibit the survival of breast cancer cells. Cancer Res 2004;64:2343-6.

34. Baselga J, Cortés J, Im SA, et al. Biomarker analyses in CLEOPATRA: a phase III, placebo-controlled study of pertuzumab in human epidermal growth factor receptor 2-positive, first-line metastatic breast cancer. J Clin Oncol 2014;32:3753-61.

35. Guarneri V, Frassoldati A, Bottini A, et al. Preoperative chemotherapy plus trastuzumab, lapatinib, or both in human epidermal growth factor receptor 2-positive operable breast cancer: results of the randomized phase II CHER-LOB study. J Clin Oncol 2012;30:1989-95.

36. Guarneri V, Dieci MV, Frassoldati A, et al. Prospective Biomarker Analysis of the Randomized CHER-LOB Study Evaluating the Dual Anti-HER2 Treatment With Trastuzumab and Lapatinib Plus Chemotherapy as Neoadjuvant Therapy for HER2-Positive Breast Cancer. Oncologist 2015;20:1001-10.

37. Loibl S, von Minckwitz G, Schneeweiss A, et al. PIK3CA mutations are associated with lower rates of pathologic complete response to anti-human epidermal growth factor receptor 2 (her2) therapy in primary HER2-overexpressing breast cancer. J Clin Oncol 2014;32:3212-20.

38. Majewski IJ, Nuciforo P, Mittempergher L, et al. PIK3CA Mutations Are Associated With Decreased Benefit to Neoadjuvant Human Epidermal Growth Factor Receptor 2-Targeted Therapies in Breast Cancer. J Clin Oncol 2015;33:1334-9.

39. Guarneri V, Dieci MV, Carbognin L, et al. $254 \mathrm{O}$ Activity of Neoadjuvant Lapatinib (L) Plus Trastuzumab (T) for Early Breast Cancer (Ebc) According to Pik3Ca Mutations: Pathological Complete Response (Pcr) Rate in the Cherlob Study and Pooled Analysis of Randomized Trials. Ann Oncol 2014;25:iv85.

40. Guerin M, Rezai K, Isambert N, et al. PIKHER2: A phase IB study evaluating buparlisib in combination with lapatinib in trastuzumab-resistant HER2-positive advanced breast cancer. Eur J Cancer 2017;86:28-36.

41. Pistilli B, Pluard T, Urruticoechea A, et al. Phase II study of buparlisib (BKM120) and trastuzumab in patients with HER2 + locally advanced or metastatic breast cancer resistant to trastuzumab-based therapy. Breast Cancer Res Treat 2018;168:357-64.

42. Loibl S, de la Pena L, Nekljudova V, et al. Neoadjuvant buparlisib plus trastuzumab and paclitaxel for women with
HER2 + primary breast cancer: A randomised, doubleblind, placebo-controlled phase II trial (NeoPHOEBE). Eur J Cancer 2017;85:133-45.

43. Jain S, Shah AN, Santa-Maria CA, et al. Phase I study of alpelisib (BYL-719) and trastuzumab emtansine (T-DM1) in HER2-positive metastatic breast cancer (MBC) after trastuzumab and taxane therapy. Breast Cancer Res Treat 2018;171:371-81.

44. Luen SJ, Salgado R, Fox S, et al. Tumour-infiltrating lymphocytes in advanced HER2-positive breast cancer treated with pertuzumab or placebo in addition to trastuzumab and docetaxel: a retrospective analysis of the CLEOPATRA study. Lancet Oncol 2017;18:52-62.

45. Solinas C, Ceppi M, Lambertini M, et al. Tumorinfiltrating lymphocytes in patients with HER2-positive breast cancer treated with neoadjuvant chemotherapy plus trastuzumab, lapatinib or their combination: A metaanalysis of randomized controlled trials. Cancer Treat Rev 2017;57:8-15.

46. Turner NC, Ro J, André F, et al. Palbociclib in HormoneReceptor-Positive Advanced Breast Cancer. N Engl J Med 2015;373:209-19.

47. Colleoni M, Montagna E. Neoadjuvant therapy for ERpositive breast cancers. Ann Oncol 2012;23 Suppl 10:x243-8.

48. McGuire WL. Estrogen Receptors in Human Breast Cancer. J Clin Invest 1973;52:73-7.

49. Rugo HS, Rumble RB, Macrae E, et al. Endocrine Therapy for Hormone Receptor-Positive Metastatic Breast Cancer: American Society of Clinical Oncology Guideline. J Clin Oncol 2016;34:3069-103.

50. Khoshnoud MR, Fornander T, Johansson H, et al. Longterm pattern of disease recurrence among patients with early-stage breast cancer according to estrogen receptor status and use of adjuvant tamoxifen. Breast Cancer Res Treat 2008;107:71-8.

51. Barnes DM, Millis RR, Gillett CE, et al. The interaction of oestrogen receptor status and pathological features with adjuvant treatment in relation to survival in patients with operable breast cancer: a retrospective study of 2660 patients. Endocr Relat Cancer 2004;11:85-96.

52. Ouyang Y, Li D, Pater JL, et al. The importance of temporal effects in evaluating the prognostic impact of joint ERPR expression in premenopausal women with node-positive breast cancer. Breast Cancer Res Treat 2005;92:115-23.

53. Yi Z, Ma F, Liu B, et al. Everolimus in hormone receptor-positive metastatic breast cancer: PIK3CA mutation H1047R was a potential efficacy biomarker in a 
retrospective study. BMC Cancer 2019;19:442.

54. Duffy MJ, Harbeck N, Nap M, et al. Clinical use of biomarkers in breast cancer: Updated guidelines from the European Group on Tumor Markers (EGTM). Eur J Cancer 2017;75:284-98.

55. Musgrove EA, Caldon CE, Barraclough J, et al. Cyclin $\mathrm{D}$ as a therapeutic target in cancer. Nat Rev Cancer 2011;11:558-72.

56. Yu Q, Sicinska E, Geng Y, et al. Requirement for CDK4 kinase function in breast cancer. Cancer Cell 2006;9:23-32.

57. Thangavel C, Dean JL, Ertel A, et al. Therapeutically activating RB: reestablishing cell cycle control in endocrine therapy-resistant breast cancer. Endocr Relat Cancer 2011;18:333-45.

58. Miller TW, Balko JM, Fox EM, et al. ER $\alpha$-dependent E2F transcription can mediate resistance to estrogen deprivation in human breast cancer. Cancer Discov 2011;1:338-51.

59. FDA. Highlights of prescribing information, abemaciclib [Internet]. [cited 2020 Nov 27]. Available online: https://www.accessdata.fda.gov/drugsatfda_docs/ label/2018/208855s000lbl.pdf

60. FDA. Highlights of prescribing information, ribociclib [Internet]. [cited 2020 Nov 27]. Available online: https://www.accessdata.fda.gov/drugsatfda_docs/ label/2017/209092s000lbl.pdf

61. Highlights of prescribing information, palbociclib [Internet]. [cited 2020 Nov 27]. Available online: https://www.accessdata.fda.gov/drugsatfda_docs/ label/2017/207103s004lbl.pdf

62. Boér K. Fulvestrant in advanced breast cancer: evidence to date and place in therapy. Ther Adv Med Oncol 2017;9:465-79.

63. Cristofanilli M, DeMichele A, Giorgetti C, et al. Predictors of prolonged benefit from palbociclib plus fulvestrant in women with endocrine-resistant hormone receptor-positive/human epidermal growth factor receptor 2-negative metastatic breast cancer in PALOMA-3. Eur J Cancer 2018;104:21-31.

64. Fribbens C, O'Leary B, Kilburn L, et al. Plasma ESR1 Mutations and the Treatment of Estrogen ReceptorPositive Advanced Breast Cancer. J Clin Oncol 2016;34:2961-8.

65. Carausu M, Bidard FC, Callens C, et al. ESR1 mutations: a new biomarker in breast cancer. Expert Rev Mol Diagn 2019;19:599-611.

66. Johnston SR, Kilburn LS, Ellis P, et al. Fulvestrant plus anastrozole or placebo versus exemestane alone after progression on non-steroidal aromatase inhibitors in postmenopausal patients with hormone-receptor-positive locally advanced or metastatic breast cancer (SoFEA): a composite, multicentre, phase 3 randomised trial. Lancet Oncol 2013;14:989-98.

67. O'Leary B, Cutts R, Huang X, et al. Genomic markers of early progression on fulvestrant with or without palbociclib for ER+ advanced breast cancer in the PALOMA-3 trial. J Clin Oncol 2019;37:1010.

68. Takeshita T, Yamamoto Y, Yamamoto-Ibusuki M, et al. Clinical significance of plasma cell-free DNA mutations in PIK3CA, AKT1, and ESR1 gene according to treatment lines in ER-positive breast cancer. Mol Cancer 2018;17:67.

69. Finn RS, Martin M, Rugo HS, et al. Palbociclib and Letrozole in Advanced Breast Cancer. N Engl J Med 2016;375:1925-36.

70. Finn RS, Liu Y, Zhu Z, et al. Biomarker Analyses of Response to Cyclin-Dependent Kinase 4/6 Inhibition and Endocrine Therapy in Women with Treatment-Naïve Metastatic Breast Cancer. Clin Cancer Res 2020; 26:110-21.

71. Finn RS, Cristofanilli M, Ettl J, et al. Treatment effect of palbociclib plus endocrine therapy by prognostic and intrinsic subtype and biomarker analysis in patients with bone-only disease: a joint analysis of PALOMA-2 and PALOMA-3 clinical trials. Breast Cancer Res Treat 2020;184:23-35.

72. André F, Ciruelos E, Rubovszky G, et al. Alpelisib for PIK3CA-Mutated, Hormone Receptor-Positive Advanced Breast Cancer. N Engl J Med 2019;380:1929-40.

73. Gul A, Leyland-Jones B, Dey N, et al. A combination of the PI3K pathway inhibitor plus cell cycle pathway inhibitor to combat endocrine resistance in hormone receptor-positive breast cancer: a genomic algorithm-based treatment approach. Am J Cancer Res 2018;8:2359-76.

74. da Silva JL, Cardoso Nunes NC, Izetti P, et al. Triple negative breast cancer: A thorough review of biomarkers. Crit Rev Oncol Hematol 2020;145:102855.

75. Cancer Genome Atlas Network. Comprehensive molecular portraits of human breast tumours. Nature 2012; 490:61-70.

76. Shah SP, Roth A, Goya R, et al. The clonal and mutational evolution spectrum of primary triple-negative breast cancers. Nature 2012;486:395-9.

77. Lehmann BD, Jovanović B, Chen X, et al. Refinement of Triple-Negative Breast Cancer Molecular Subtypes: Implications for Neoadjuvant Chemotherapy Selection. 
PLoS One 2016;11:e0157368.

78. Kim JY, Park K, Jung HH, et al. Association between Mutation and Expression of TP53 as a Potential Prognostic Marker of Triple-Negative Breast Cancer. Cancer Res Treat 2016;48:1338-50.

79. Bae SY, Lee JE, Lee SK, et al. Immunohistochemical status of $\mathrm{p} 53$ as prognostic factor in patients with node negative triple-negative breast cancer. Ann Oncol 2016;27:vi52.

80. Synnott NC, Murray A, McGowan PM, et al. Mutant p53: a novel target for the treatment of patients with triplenegative breast cancer? Int J Cancer 2017;140:234-46.

81. Viale G, Regan MM, Mastropasqua MG, et al. Predictive Value of Tumor Ki-67 Expression in Two Randomized Trials of Adjuvant Chemoendocrine Therapy for NodeNegative Breast Cancer. JNCI J Natl Cancer Inst 2008;100:207-12.

82. Kulkarni Y. Reversing Resistance to PARP-Inhibitor in Triple Negative Breast Cancer. [cited 2020 Nov 30]; Available online: https://grantome.com/grant/NIH/SC3GM122655-03

83. McCann KE, Hurvitz SA. Advances in the use of PARP inhibitor therapy for breast cancer. Drugs Context 2018;7:212540.

84. Robson M, Im SA, Senkus E, et al. Olaparib for Metastatic Breast Cancer in Patients with a Germline BRCA Mutation. N Engl J Med 2017;377:523-33.

85. Fasching PA, Hu C, Hart S, et al. Germline BRCA1 and BRCA2 mutations in patients with HER2-negative metastatic breast cancer $(\mathrm{mBC})$ treated with first-line chemotherapy: Data from the German PRAEGNANT registry. J Clin Oncol 2019;37:1048.

86. Eikesdal HP, Yndestad S, Blix ES, et al. Neoadjuvant olaparib monotherapy in primary triple negative breast cancer. Ann Oncol 2019;30:v60.

87. Schmid P, Rugo HS, Adams S, et al. Atezolizumab plus nab-paclitaxel as first-line treatment for unresectable, locally advanced or metastatic triple-negative breast cancer (IMpassion 130): updated efficacy results from a randomised, double-blind, placebo-controlled, phase 3

doi: $10.21037 /$ tbcr-20-66

Cite this article as: He J, Jia Y. The evolution and advances of biomarker use in clinical trials for breast cancer treatment-a narrative review. Transl Breast Cancer Res 2021;2:6. trial. Lancet Oncol 2020;21:44-59.

88. Emens LA, Loi S, Rugo HS, et al. Abstract GS1-04: IMpassion130: Efficacy in immune biomarker subgroups from the global, randomized, double-blind, placebocontrolled, phase III study of atezolizumab + nab-paclitaxel in patients with treatment-naïve, locally advanced or metastatic triple-negative breast cancer. Cancer Res 2019;79:GS1-04-GS1-04.

89. Emens LA, Cruz C, Eder JP, et al. Long-term Clinical Outcomes and Biomarker Analyses of Atezolizumab Therapy for Patients With Metastatic Triple-Negative Breast Cancer: A Phase 1 Study. JAMA Oncol 2019;5:74-82.

90. Adams S, Loi S, Toppmeyer D, et al. Pembrolizumab monotherapy for previously untreated, PD-L1-positive, metastatic triple-negative breast cancer: cohort B of the phase II KEYNOTE-086 study. Ann Oncol 2019;30:405-11.

91. Schmid P, Abraham J, Chan S, et al. Capivasertib Plus Paclitaxel Versus Placebo Plus Paclitaxel As First-Line Therapy for Metastatic Triple-Negative Breast Cancer: The PAKT Trial. J Clin Oncol 2020;38:423-33.

92. Kim SB, Dent R, Im SA, et al. Ipatasertib plus paclitaxel versus placebo plus paclitaxel as first-line therapy for metastatic triple-negative breast cancer (LOTUS): a multicentre, randomised, double-blind, placebocontrolled, phase 2 trial. Lancet Oncol 2017;18:1360-72.

93. Coombes RC, Page K, Salari R, et al. Personalized Detection of Circulating Tumor DNA Antedates Breast Cancer Metastatic Recurrence. Clin Cancer Res 2019;25:4255-63.

94. O’Leary B, Hrebien S, Morden JP, et al. Early circulating tumor DNA dynamics and clonal selection with palbociclib and fulvestrant for breast cancer. Nat Commun 2018;9:896.

95. Guarneri V, Dieci MV, Bisagni G, et al. De-escalated therapy for HR+/HER2+ breast cancer patients with Ki67 response after 2-week letrozole: results of the PerELISA neoadjuvant study. Ann Oncol 2019;30:921-6. 